\title{
The homolateral simultaneous pancreas-kidney transplantation: a single-center experience in China
}

\author{
Lei Zhang ${ }^{1,2}$, Zheng Chen ${ }^{1,2}$, Xingqiang Lai ${ }^{1}$, Junjie Ma ${ }^{1}$, Jiali Fang ${ }^{1}$, Yuhe Guo ${ }^{1}$, Guanghui $\mathrm{Li}^{1}, \mathrm{Lu} \mathrm{Xu}^{1}$, \\ Wei Yin ${ }^{1}$, Yunyi Xiong ${ }^{1}$, Luhao Liu ${ }^{1}$, Rongxin $\mathrm{Chen}^{1}, \mathrm{Li} \mathrm{Li}^{1}$ \\ ${ }^{1}$ Department of Organ Transplantation, Second Affiliated Hospital of Guangzhou Medical University, Guangzhou 510260, China; ${ }^{2}$ Sino-French \\ Hoffmann Institute, Guangzhou Medical University, Guangzhou 511436, China \\ Contributions: (I) Conception and design: Z Chen, L Zhang; (II) Administrative support: Z Chen; (III) Provision of study materials or patients: X \\ Lai, J Ma, R Chen, L Li; (IV) Collection and assembly of data: L Xu, W Yin, Y Xiong, L Liu, J Fang, G Li; (V) Data analysis and interpretation: L \\ Zhang, Y Guo; (VI) Manuscript writing: All authors; (VII) Final approval of manuscript: All authors. \\ Correspondence to: Zheng Chen. Department of Organ Transplantation, Second Affiliated Hospital of Guangzhou Medical University, Guangzhou \\ 510260, China. Email: docchenzheng@163.com.
}

\begin{abstract}
Background: To preliminarily explore the clinical effect of the homolateral simultaneous pancreas and kidney (SPK) transplantation in China.

Methods: SPK using the surgical technique was performed in 88 patients from September 2016 to July 2019 in the Department of Transplantation of the Second Affiliated Hospital of Guangzhou Medical University. Patients were followed up for 2 to 36 months to summarize the efficacy and complications.

Results: Up to now, 83 patients have achieved good clinical efficacy with no major surgical complications, but 3 patients died of severe infection and 2 have graft loss. The serum creatinine (Scr) at 1, 3, 6, 12, 24 months after operation were 118, 119, 116, 114, $110 \mathrm{umol} / \mathrm{L}$; fasting blood glucose were 5.8, 5.0, 5.0, 4.9, $4.8 \mathrm{mmol} / \mathrm{L}$; and glycated hemoglobin at 3, 6, 12, 24 months after transplantation were 5.2\%, 5.5\%, 5.2\%, $5.1 \%$. One- and 2-year patient, pancreas, and kidney graft survival rates were $96.1 \%, 93.8 \%, 95.0 \%$ and $96.1 \%, 93.8 \%$, 95.0\%. Main complications included 20 cases of kidney rejection $(22.7 \%)$, 22 cases of pancreas rejection $(25.0 \%), 31$ cases of pulmonary infection (35.2\%), 28 cases of gastrointestinal bleeding (31.8\%), 2 cases of splenic vein thrombosis $(2.3 \%), 2$ case of artery thrombosis and anastomotic leak $(2.3 \%)$ and 2 cases of pancreas allograft dysfunction (2.3\%).
\end{abstract}

Conclusions: homolateral simultaneous pancreas-kidney transplantation has a definite therapeutic effect. The relatively simple surgical method can be done with a smaller wound in unilateral fossa iliaca.

Keywords: Simultaneous pancreas and kidney transplantation (SPK transplantation); renal function; fasting glucose; complications

Submitted Sep 29, 2019. Accepted for publication Oct 25, 2019.

doi: 10.21037/atm.2019.10.117

View this article at: http://dx.doi.org/10.21037/atm.2019.10.117

\section{Introduction}

Diabetes is a global disease, with a prevalence rate of $7.2 \%$ in China. It is estimated that by 2030, there will be 366 million patients with diabetes worldwide and over 100 million people with diabetic nephropathy (1). Simultaneous pancreas and kidney (SPK) transplantation have been widely accepted as an effective therapeutic approach to patients with endstage diabetic nephropathy and is performed mostly and maturely in multi-organ transplantation. Kelly et al. performed the first pancreas transplantation in 1966 (2), by 2015 , more than 40,000 pancreas transplantation have been performed worldwide. Nevertheless, only several Chinese organ transplant centers carry out SPK because of surgery complications. In 2016, our center began to adopt the modified SPK technology to carry out the treatment of diabetic nephropathy. In this study, the preliminary 


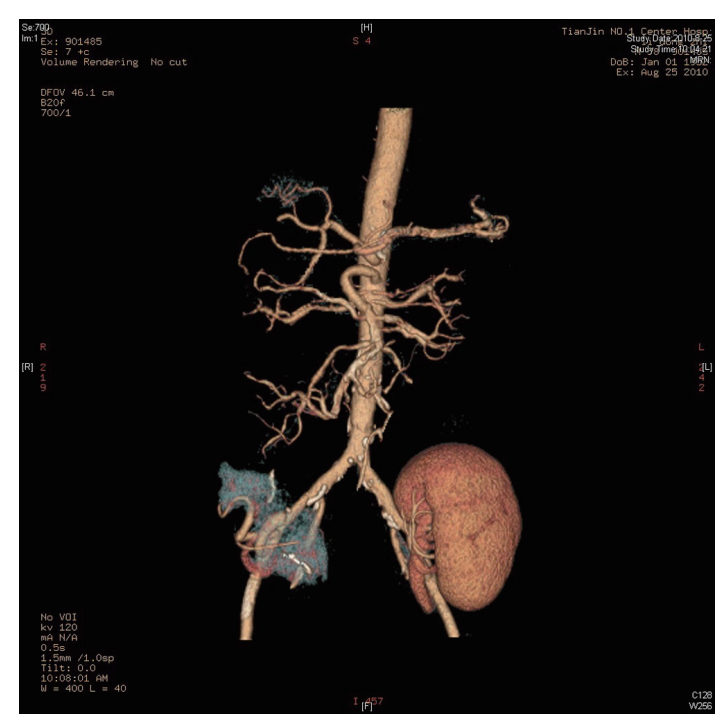

Figure 1 Pancreas and kidney placement in the bilateral iliac fossa.

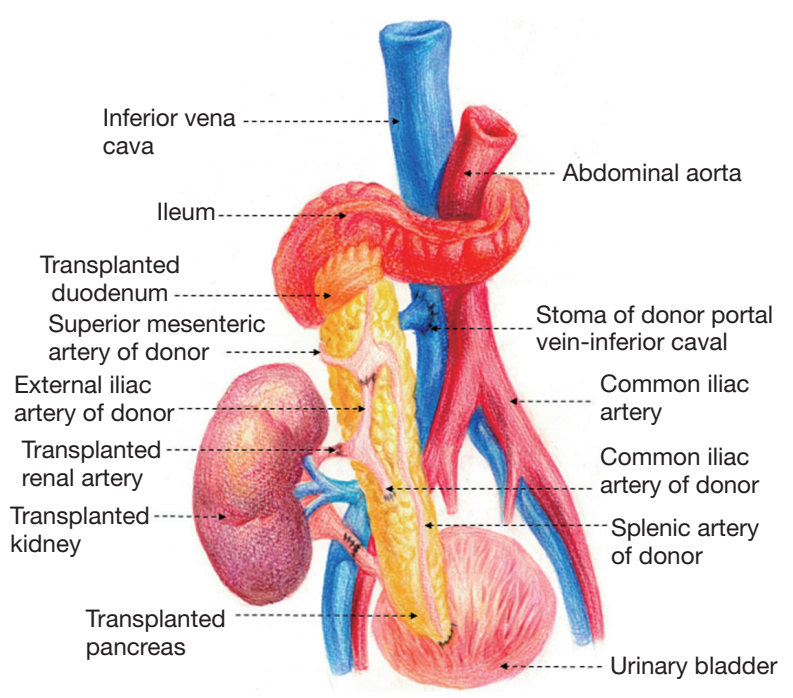

Figure 2 Pancreatic and renal grafts placed in the same iliac fossa, using systemic venous endocrine drainage.

treatment results are summarized to promote the further development of this work in China.

\section{Methods}

\section{Clinical data}

All cases involved in this study were approved by the hospital ethics committee. Between September 2016 and July 2019, a total of 88 SPK transplantation were performed, with a mean follow-up of 13 (range, 2-36) months. Eightyeight patients (70 males, 18 females) were aged from 27 to 67 , duration of dialysis from 0 to 83 months. The primary disease was diabetic nephropathy, including 13 cases of type I diabetes and 75 cases of type II diabetes. Both organs the pancreas and the kidney are procured from a donor after brain death (DBD), with mean donor age of 31.2 \pm 9.9 (range, 10-48) years old. Mean creatinine before organ acquisition was $116.8 \pm 54.6 \mu \mathrm{mol} / \mathrm{L}$, mean blood glucose under stress was $8.8 \pm 4.0 \mathrm{mmol} / \mathrm{L}$, mean glycosylated hemoglobin was $(5.3 \pm 0.4) \%$, and mean body mass index was $22.8 \pm 5.4 \mathrm{~kg} / \mathrm{m}^{2}$.

\section{Surgical techniques and immunosuppression}

\section{Surgical techniques}

The surgical techniques of SPK transplantation have been developing and improving. Up to now, Pancreas and kidney placement in the bilateral iliac fossa, as shown in Figure 1, is the most commonly used technique in many transplant centers, and several mature surgical techniques have been formed (3). There are two main differences, one is internal secretion drainage of the pancreas via the vena cava venous or the portal vein system, and another is external secretion drainage via intestinal tract or bladder (4). In 2003, The Emory Transplant Center reported a new technique for SPK transplantation utilizing a single arterial conduit to vascularize both organs, with pancreatic and renal allograft placed in the same iliac fossa (5). Later, the surgical technique underwent another evolution, in which the pancreatic portal vein was anastomosed with the end to the side of the recipient inferior vena cava to avoid the free recipient portal vein (Figures 2,3) (6). Herein, we describe our experience with this technique for SPK transplantation.

The donor's whole abdominal organs were obtained, with preservation of the entire duodenum and segment of the jejunum with the allograft. The donor's one side of the common, internal, and external iliac artery (Y graft) were collected.

First, during back table preparation of the left kidney, the renal artery was sewn in an end-to-end fashion to the internal iliac limb of the donor iliac artery Y graft. The common iliac artery and external iliac artery were reserved (Figure 4).

Repair processes in the pancreas are the most crucial step in SPK transplantation. The donor's pancreas, duodenum and arterial system of the pancreas including the celiac trunk, common hepatic artery, proper hepatic artery, splenic 


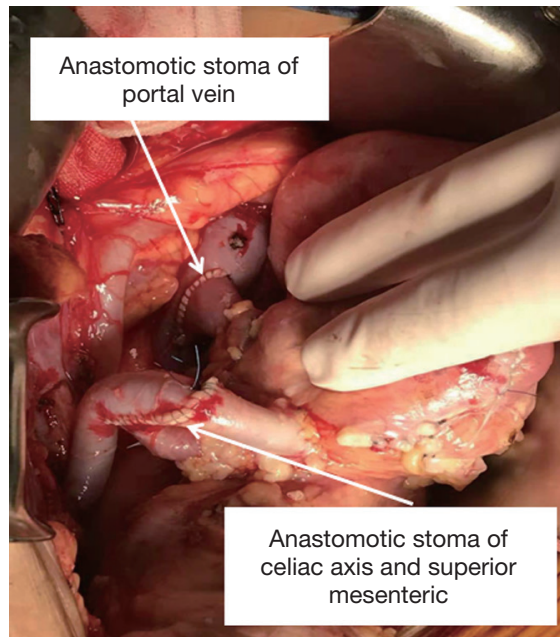

Figure 3 Anastomotic stoma of portal vein and artery.

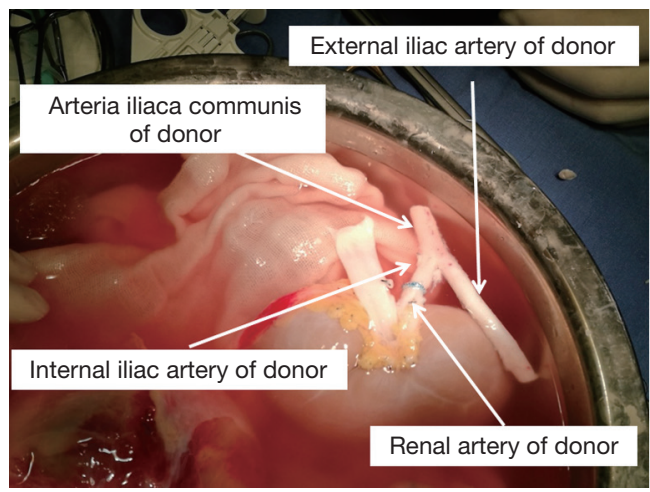

Figure 4 Renal reconstruction.

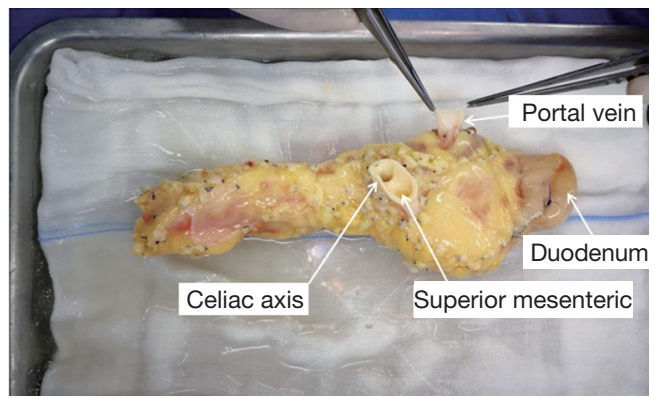

Figure 5 Pancreatic reconstruction.

artery, gastroduodenal artery, and superior mesenteric artery were obtained, which was super complicated and highly variable. The length of the portal vein was about 1 to $1.5 \mathrm{~cm}$, the abdominal aortic sleeve plates of the celiac trunk and the superior mesenteric artery were reserved for anastomoses, and the duodenal length was about 8 to $10 \mathrm{~cm}$ for anastomoses, as shown in Figure 5.

We entered the abdominal cavity through the recipient's right lateral transrectus incision. The appendix was routinely removed, and after the lateral peritoneum was opened, completed kidney transplantation first. The kidney was then revascularized by sewing the common iliac limb of the $\mathrm{Y}$ graft to the limited pliable segment of the recipient's right external iliac artery, the renal vein to the right external iliac vein, and end-to-end donor's ureter to recipient's ureter anastomosis. We used the method creatively for urinary tract reconstruction in SPK. This technique allowed SPK transplantation without free recipient's bladder, which shrank the notch and reduced the difficulty of operation to a certain extent. After opening the renal vessels, the renal graft was placed in the right iliac fossa, the lateral peritoneum was partially closed, and external iliac limb of the $\mathrm{Y}$ graft was exposed in the abdominal cavity for later use. The next step was to perform pancreas transplantation, end-to-side anastomosis of the pancreatic portal vein and inferior vena cava, end-to-end anastomosis of the common flap sleeve piece of the abdominal trunk and superior mesenteric artery with the previously reserved external iliac limb of the Y graft, side-to-side anastomosis of the duodenum and the recipient ileum, and anastomosis of the anastomotic site about 60 to $70 \mathrm{~cm}$ from the ileocecal part (as shown in Figure 3).

\section{Immunosuppressive regimens}

Polyclonal antibody and monoclonal antibody induction therapy were selected. Preoperative intravenous infusion of methylprednisolone (MP) was 250 to $500 \mathrm{mg}$, while day 1 , 2 and 3 post-operation required a dose of 500, 250, and $250 \mathrm{mg}$, respectively. For maintenance therapy, all SPK patients received tacrolimus (FK506), mycophenolate mofetil (MMF) and steroids (prednisone), with a daily dose of $0.05-01 \mathrm{mg} / \mathrm{kg}$ in FK506, and $1-1.5 \mathrm{~g}$ in MMF. The dosage was adjusted according to FKS06 valley concentration (C0). Prednisone was taken orally $30 \mathrm{mg}$ at day 4 after surgery, with a reduction of $5 \mathrm{mg}$ every 7 days until a maintenance dose of 10 or $5 \mathrm{mg}$.

\section{Observation index and definitions}

Acute rejection (AR) and delayed graft function (DGF)

Rejection of the renal graft was diagnosed by laboratory 


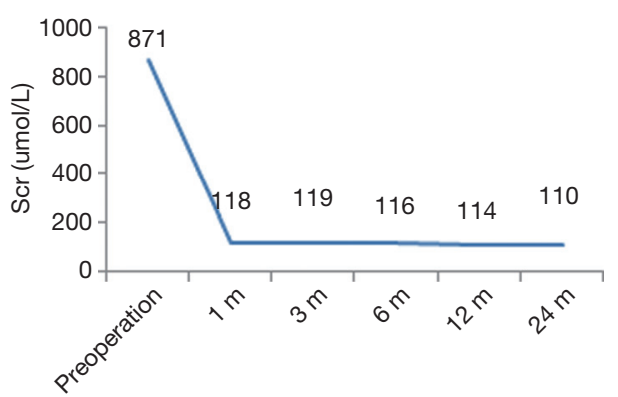

Figure 6 Postoperative Scr. Scr, serum creatinine; m, month.

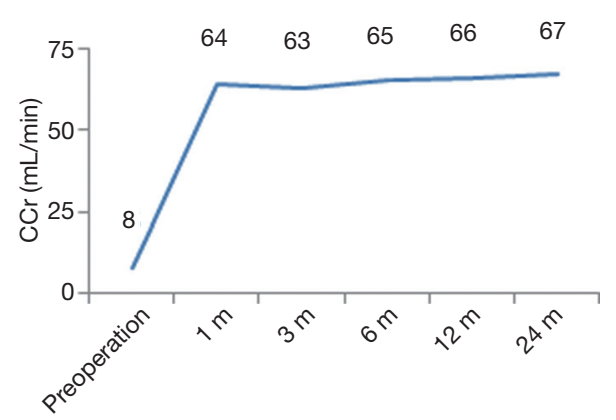

Figure 7 Postoperative CCr. CCr, creatinine clearance rate; m, month.

examination, ultrasound examination, and biopsy of a transplanted kidney (Banff grade IA or greater) and so on. The pancreas rejection was diagnosed by serum lipase, hematuria amylase, pancreatic ultrasound, and blood glucose. Once diagnosed, $250 \mathrm{mg} /$ day of MP was used for 3 days. Steroids-resistant patients were given to antithymocyte globulin 50 to $75 \mathrm{mg} / \mathrm{d}$ or anti-lymphocyte globulin (ATG) $100 \mathrm{mg} / \mathrm{d}$ for 3 to 7 days, as appropriate. However, in severe cases, the patient was treated with anti-CD20 antibodies (rituximab) 400 to $500 \mathrm{mg}$ and human immunoglobulin. Renal allograft DGF was defined as requiring hemodialysis at least once within one week of transplantation (7).

\section{Patient survival \& pancreas graft survival \& renal graft survival}

Renal graft survival was defined as the exclusion of graft resection, restoration of dialysis, and death with graft function. Survival of the transplanted pancreas excluded resection of the transplanted pancreas, recovery of preoperative insulin dosage and death with graft function.

\section{Renal allograft function}

Creatinine clearance rate $(\mathrm{CCr})$ was calculated by the
Cockcroft-Gault equation to evaluate renal allograft function. At present, serum creatinine (Scr) is used in all transplant centers to reflect renal function directly, so Scr is also provided as reference. The recording time was $1,3,6$, 12 , and 24 months after transplantation.

\section{Pancreas allograft function}

Fasting blood glucose, glycosylated hemoglobin, fasting C-peptide, fasting insulin and other laboratory indicators single-center for evaluation, but reuse of insulin and the dosage was not as same as preoperative was defined as pancreas allograft dysfunction.

\section{Follow-up endpoints}

(I) Renal graft loss and pancreas graft loss;

(II) Death in patients.

\section{Statistical analysis}

Data were analyzed with SPSS 13.0. The continuity data were expressed by mean \pm standard deviation (SD). Patient, pancreatic, and kidney survival rate were accomplished by Kaplan-Meier survival analysis method. The test level for all statistical methods was $\alpha=0.05$.

\section{Results}

\section{Renal graft function}

Except for one patient with DGF, the graft function of the other patients recovered successfully. Postoperative Scr and CCr were shown in Figures 6,7.

\section{Pancreas graft function}

Most SPK recipients reduced their blood glucose level to normal within 2 to 3 days post-transplantation, with the independence of insulin. Two patients received insulin again due to high postoperative blood glucose, but the dose was much lower than the preoperative level. Data of fasting blood glucose, fasting insulin, fasting $\mathrm{C}$-peptide and glycosylated hemoglobin were shown in Figure 8.

\section{Complications}

Complications related to the SPK transplantation were medical complications (Table 1), with a higher frequency of infection and rejection. After active treatment, all the 

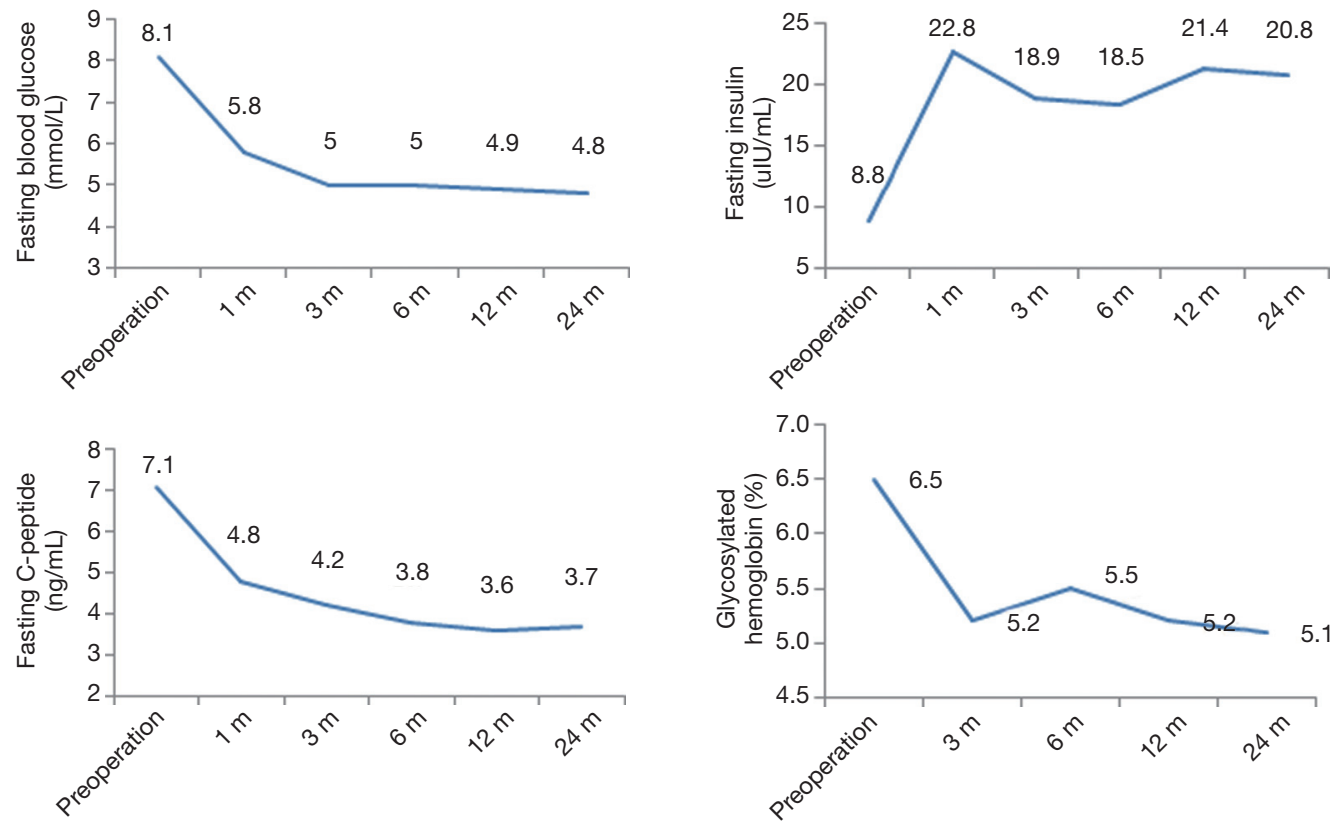

Figure 8 Data of pancreas graft function including fasting blood glucose, fasting insulin, fasting C-peptide and glycosylated hemoglobin post-operation. $\mathrm{m}$, month.

Table 1 Complications in our center of the 88 cases

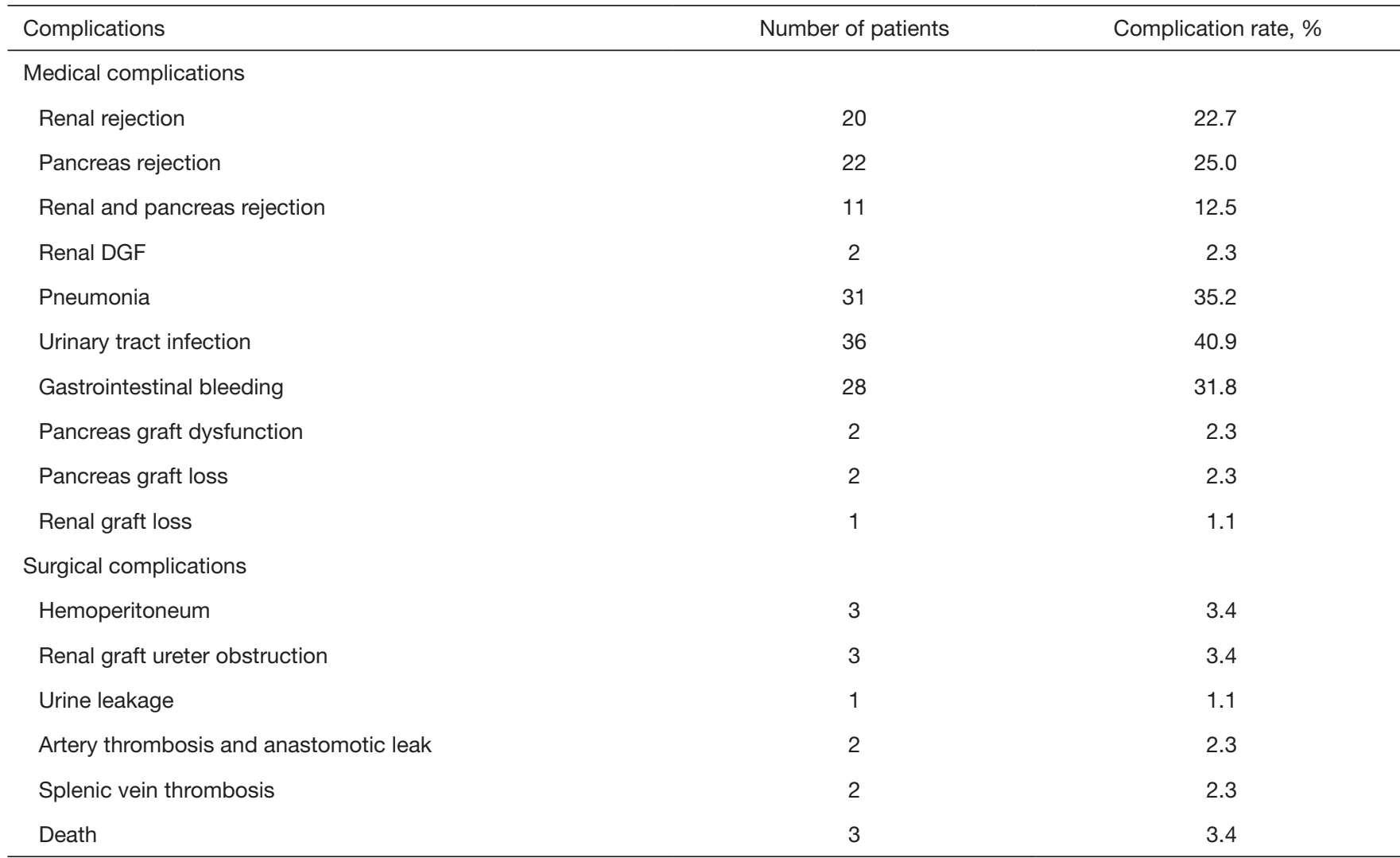

DGF, delayed graft function. 
medical complications were cured except 2 patients died of severe pneumonia. The two recipients of splenic venous thrombosis successfully treated the transplanted pancreas after treatment of low molecular heparin and standard anticoagulant therapy of rivaroxaban, showing good function. While the incidence of surgical complications, especially severe surgical complications, were controlled ideally, which were not more than other transplant centers $(3,8)$. All surgical complications were satisfactorily treated except 2 cases of artery thrombosis and subsequent anastomotic leak who received excision of the pancreas; even so, one receptor died of a severe abdominal infection after reoperation (Figure 9).

\section{Patient, pancreas graft and renal graft survival rate at 2 to 36 months after transplantation}

At the endpoint of follow-up, 3 recipients died, all of them male. The first patient, 54 years old, died at 59 days after the operation due to renal DGF, AR, pulmonary infection, and gastrointestinal bleeding. The second case was 46 years old, with severe pneumonia and gastrointestinal bleeding, and died at 10 months post-transplantation. The third case died at 1 month because of intestinal anastomotic leak and severe infection. All the other patients were followed up regularly, and no patients were lost to follow-up.

Patient, pancreas graft and renal graft survival rate at 1 and 2 years after SPK were 96.1\%, 93.8\%, 95.0\% and $96.1 \%$, 93.8\% , 95.0\%, analyzed by Kaplan-Meier ratio (Figure 10).

\section{Discussion}

SPK transplantation is the first option for patients with end-stage diabetic nephropathy and is performed mostly and maturely in multi-organ transplantation (9). In the 1990s, with the improvement of surgical techniques and the development of immunosuppressive agents, pancreas transplantation was widely carried out and became the gold standard for the treatment of end-stage diabetes. SPK transplantation has gradually become the best treatment for end-stage diabetic nephropathy. SPK transplantation can improve a patient's quality of life, prolong the survival period of patients with diabetic nephropathy, prevent and reverse the complications of diabetes, and have a better survival rate and graft half-life than simply kidney transplantation. For type II diabetes, pancreas transplantation has similar long-term effects as type I diabetes $(10,11)$. After 50 years

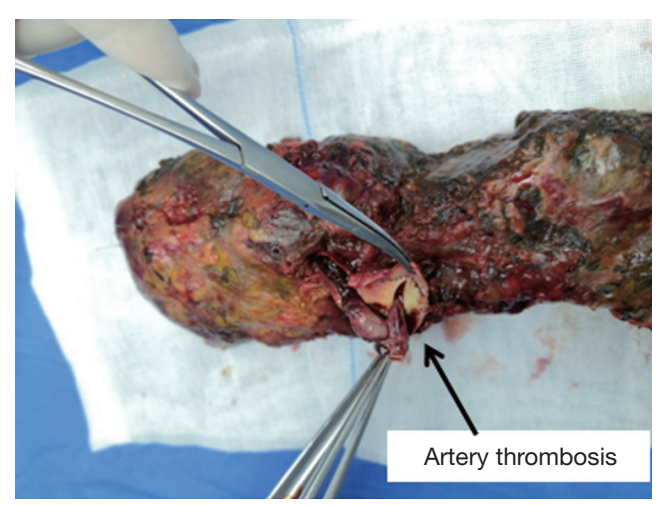

Figure 9 Excision of pancreas graft because of artery thrombosis and pancreas infarct.

of development, SPK transplantation has reached maturity. Statistically, the 1-, 10- and 15-year survival rates of the patients in SPK transplantation reached or were close to $94 \%, 70 \%$, and $56 \%$, respectively, and the pancreas graft survival rates were $86 \%, 53 \%$, and $36 \%$, and the kidney graft survival rates were $95 \%, 60 \%$ and $40 \%(12,13)$.

SPK transplantation started late in China. In 1982 and 1989, Tongji Hospital of Tongji Medical College of HUST respectively carried out the first pancreas transplantation and SPK transplantation in China. But in recent 20 years, only several hospitals have carried out pancreas transplantation successively (14). So, the total number of pancreas transplantation in China is less than 500, compared to less than $1 \%$ of the total number of pancreas transplantation in the world.

Surgical complications are the leading cause of failure in the combined pancreas and kidney transplantation. So much as in $80 \%$ of cases requiring further open surgery, the transplanted pancreas should be removed in the early stage (15). According to the statistics of the University of Minnesota, which initiated SPK transplantation, the reoperation rate was $36 \%$, and even as high as $50 \%$ in some centers (16), including arteriovenous thrombosis (6-17\%), local or diffuse abdominal tissue necrosis $(12 \%)$, pancreatitis (3-12\%), abdominal infection (1-5\%), anastomotic leakage $(0.5-2 \%)$ and abdominal hematoma $(0.5 \%)$, and so on (17-19). The most common complications leading to pancreatectomy and death were splenic vein thrombosis and abdominal tissue necrosis, respectively (17).

Because of this, the continuous improvement of surgical techniques and surgical methods is especially important. The differences in the operation are in three aspects. First, endocrine drainage, the advantages of choosing portal 

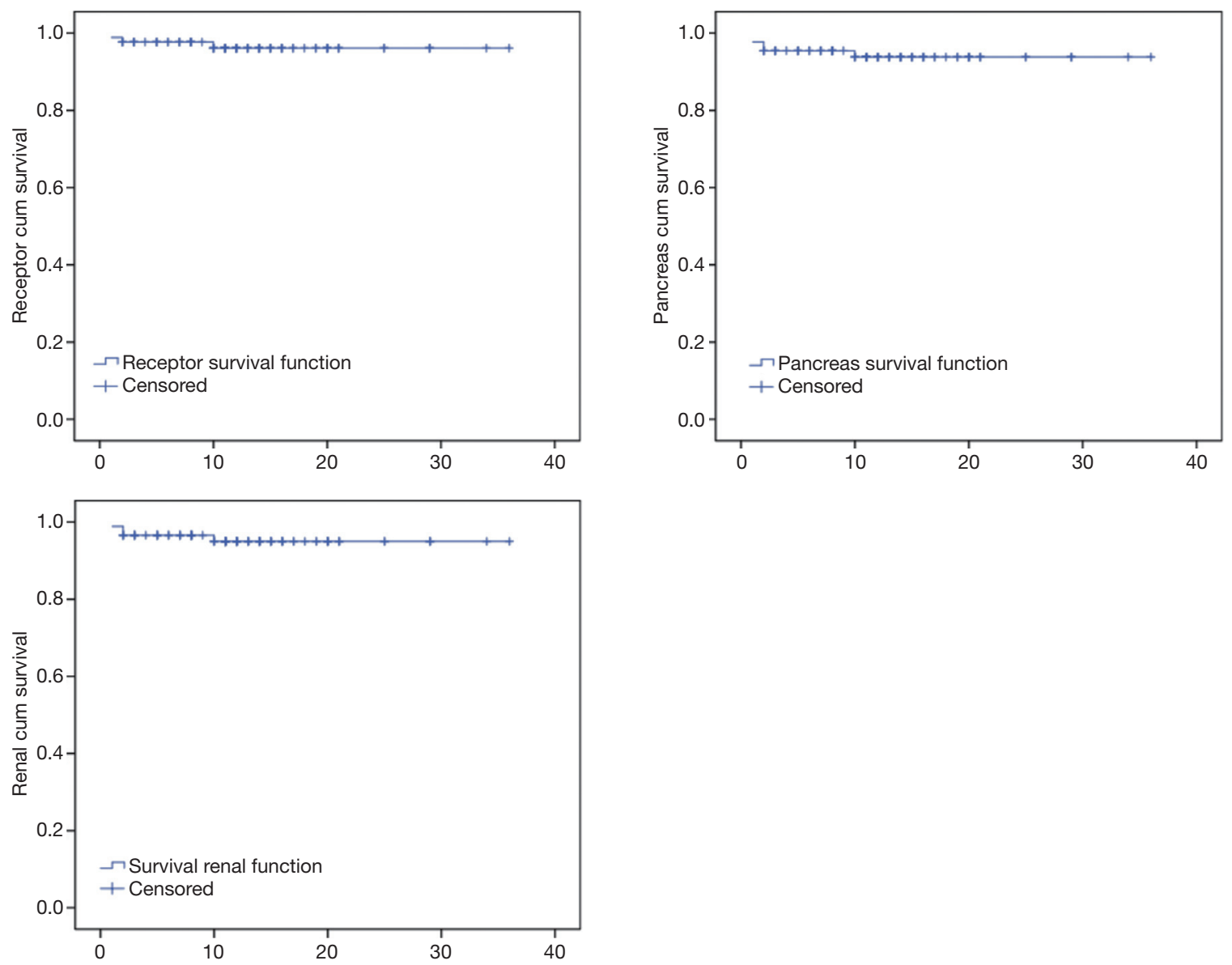

Figure 10 Patient, pancreas graft and renal graft survival rate at 1 and 2 years after SPK. SPK, simultaneous pancreas and kidney.

venous drainage (PVD) is in line with the physiological drainage mode, while the disadvantage is that it would increase the difficulty of surgery and the probability of complications. Compared to PVD, systemic venous drainage (SVD) has a set of advantages and disadvantages theoretically. However, in our observation, we did not find any obvious deficiency of SVD in the physiological function and metabolism of insulin, so we prefer SVD. Second, exocrine drainage. Although bladder drainage became the mainstream within a period, the choice of enteric drainage was more consistent with the characteristics of the anatomical structure than that of bladder drainage, reducing the bladder complications. Therefore, it was adopted by most centers in recent years, including our center. Thirdly, the location of the pancreas and kidney. Compared with the previous surgical techniques in which the pancreas and kidney were separated into two sides of the iliac fossa, the advantages of the modified SPK transplantation lie in the simplification of the operation mode, the reduction of the perfusion pressure of the pancreas, a low-perfusion organ, and the preservation of one side of the iliac blood vessel for the patient's retransplantation if possibly.

After adopting the technique, our center controlled serious surgical complications, preferably in patients with SPK transplantation, with an incidence rate of $2.3 \%$ of and the same incidence of bowel anastomotic leak. After active anticoagulant treatment, the 2 patients of splenic vein thrombosis were no need to remove the transplanted pancreas. But the prognosis of bowel anastomotic leak is not ideal, and one case has to excision of the pancreas, and another died. What should be specially mentioned is our ureteral complication. After creatively improving anastomosis method of the ureter in SPK, the incidence rate of ureteral complication was only $4.5 \%$ including ureter obstruction and Urine leakage with the smaller notch and simpler operation. 
Our experience in the new surgical technique of SPK transplantation is summarized as follows. Firstly, it is a crucial step to fully expose the abdominal cavity to establish a good operative space. Second, repair of the pancreas is the key to the success of the surgery. The pancreatic capsule must be kept intact, and the peripancreatic tissue should be ligated as far as possible. Meanwhile, the appropriate degree of looseness after ligation of the splenic artery and splenic vein should be maintained to avoid the occurrence of splenic arteriovenous thrombosis after mechanical compression. Thirdly, keep duodenum of appropriate length, or intestinal necrosis and intestinal leakage may occur if it is too long. Fourth, the portal vein should be 1 to $1.5 \mathrm{~cm}$ to avoid portal vein thrombosis. Fifth, when repaired the donor iliac artery, the longer external iliac artery and the shorter internal iliac artery should be retained as much as possible. Sixth, Patients with diabetes must be evaluated for iliac artery status. Lastly, the abdominal CT of the recipients must be carefully read before the operation, for in our center, two recipients' inferior vena cava was transposed to the left of the abdominal aorta.

Medical complications are still a problem in SPK transplantation that cannot be ignored. In medical complications, the incidence of AR is higher. Using immune-inducing drugs similar to those used in simple kidney transplantation, the renal allograft rejection rate of our SPK transplantation recipients was $22.7 \%$, the pancreas allograft rejection rate was $25 \%$, and rejection rate of renal and pancreas allograft was $12.5 \%$, which was obviously higher than that of the incidence of simple renal transplantation (20). It was not consistent with the view that AR rate in multiple organ transplantation would decrease. Some foreign studies have similar conclusions with ours (21). It should be emphasized that the incidence rate of gastrointestinal bleeding was $31.8 \%$ in our center, and it usually occurred at 7 to 10 days after transplantation. We know that the intestinal tract is an organ prone to rejection. Whether this phenomenon is simple local bleeding at the intestinal anastomosis or a duodenal rejection after transplantation, due to the temporary technical means, it is impossible to obtain pathological specimens for accurate judgment, but we prefer the latter. In addition to the active hemostasis therapy, the gastrointestinal bleeding of these patients was effectively controlled after anti-rejection therapy. Further studies on the AR of SPK transplantation are still needed.

In conclusion, with the new surgical techniques of SPK transplantation, the function of all organs recovered well, and no DGF of the transplanted pancreas was yet to be seen. The blood glucose of all patients was rapidly reduced to normal, and the postoperative quality of life of the patients was ideal. However, the incidence of AR and infection in the early postoperative period is high and should be carefully observed and treated in time. How to reduce the early postoperative infection rate and the death rate of patients caused by medical complications is also the key content that we need to summarize further and improve. We will also accumulate more experience to carry out the SPK transplantation better to benefit more patients with diabetes mellitus and renal insufficiency.

\section{Acknowledgments}

We would like to thank the patients for their support in permitting us to publish the findings of our research.

Funding: The work was supported by Scientific Research Projects of Universities administered by Guangzhou (grant no.1201630620) and Guangdong Province Science and Technology Department (grant no.2015B02-0226002). We also have received funding from Sino-French Hoffmann Institute of Guangzhou Medical University.

\section{Footnote}

Conflicts of Interest: The authors have no conflicts of interest to declare.

Ethical Statement: The authors are responsible for all aspects of the work in ensuring that questions related to the accuracy or integrity of any part of the work are appropriately investigated and resolved. This study was conducted in accordance with the Declaration of Helsinki and approved by Chinese Ethics Committee of Registering Clinical Trials (No. ChiCTR1900026543). Informed consent was obtained from all patients for their dates to be used for research.

\section{References}

1. Reutens AT, Atkins RC. Epidemiology of diabetic nephropathy. Contrib Nephrol 2011;170:1-7.

2. Kelly WD, Lillehei RC, Merkel FK, et al. Allotransplantation of the pancreas and duodenum along with the kidney in diabetic nephropathy. Surgery 1967;61:827-37.

3. Ming C, Luo X, Gong N, et al. Long-term follow-up of 
53 cases of combined pancreas-kidney transplantation. Chinese Journal of Organ Transplantation 2012;33:523-7.

4. Laftavi MR, Gruessner A, Gruessner R. Surgery of pancreas transplantation. Curr Opin Organ Transplant 2017;22:389-97.

5. Tso PL, Cash MP, Pearson TC, et al. Simultaneous pancreas-kidney transplantation utilizing a common arterial conduit: early experience and potential applications. Am J Transplant 2003;3:1440-3.

6. Fridell JA, Shah A, Milgrom ML, et al. Ipsilateral placement of simultaneous pancreas and kidney allografts. Transplantation 2004;78:1074-6.

7. Stratta RJ, Rohr MS, Sundberg AK, et al. Intermediateterm outcomes with expanded criteria deceased donors in kidney transplantation: a spectrum or specter of quality? Ann Surg 2006;243:594-601; discussion 601-3.

8. O'Malley RB, Moshiri M, Osman S, et al. Imaging of pancreas transplantation and its complications. Radiol Clin North Am 2016;54:251-66.

9. Redfield RR, Scalea JR, Odorico JS. Simultaneous pancreas and kidney transplantation: current trends and future directions. Curr Opin Organ Transplant 2015;20:94-102.

10. Sampaio MS, Kuo HT, Bunnapradist S. Outcomes of simultaneous pancreas-kidney transplantation in type 2 diabetic recipients. Clin J Am Soc Nephrol 2011;6:1198-206.

11. Light J, Tucker M. Simultaneous pancreas kidney transplants in diabetic patients with end-stage renal disease: the 20-yr experience. Clin Transplant 2013;27:E256-63.

12. McCullough KP, Keith DS, Meyer KH, et al. Kidney and pancreas transplantation in the United States, 19982007: access for patients with diabetes and end-stage renal disease. Am J Transplant 2009;9:894-906.

13. Ziaja J, Chudek J, Kolonko A, et al. Does simultaneously

Cite this article as: Zhang L, Chen Z, Lai X, Ma J, Fang J, Guo Y, Li G, Xu L, Yin W, Xiong Y, Liu L, Chen R, Li L. The homolateral simultaneous pancreas-kidney transplantation: a single-center experience in China. Ann Transl Med 2019;7(22):629. doi: 10.21037/atm.2019.10.117 transplanted pancreas improve long-term outcome of kidney transplantation in type 1 diabetic recipients? Transplant Proc 2011;43:3097-101.

14. Zheng J, Song W, Jinpeng TU, et al. Surgical treatment of simultaneous pancreas-kidney transplantation: causes and outcomes. Chinese Journal of Organ Transplantation 2014;35:724-7.

15. Khubutia MS, Pinchuk AV, Dmitriev IV, et al. Surgical complications after simultaneous pancreas-kidney transplantation: a single-center experience. Asian J Surg 2016;39:232-7.

16. Chan CM, Chim TM, Leung KC, et al. Simultaneous pancreas and kidney transplantation as the standard surgical treatment for diabetes mellitus patients with endstage renal disease. Hong Kong Med J 2016;22:62-9.

17. Grochowiecki T, Gałązka Z, Madej K, et al. Surgical complications related to transplanted pancreas after simultaneous pancreas and kidney transplantation. Transplant Proc 2014;46:2818-21.

18. Malaise J, Steurer W, Koenigsrainer A, et al. Simultaneous pancreas-kidney transplantation in a large multicenter study: surgical complications. Transplant Proc 2005;37:2859-60.

19. Gruessner AC, Sutherland DE, Gruessner RW. Pancreas transplantation in the United States: a review. Curr Opin Organ Transplant 2010;15:93-101.

20. Zhang L, Chen Z, Ma J, et al. Clinical follow-up study of curative effect for kidney transplant recipients between two different sources of donors. J Clin Urology 2016;31:466-9.

21. Samoylova ML, Borle D, Ravindra KV. Pancreas transplantation: indications, techniques, and outcomes. Surg Clin North Am 2019;99:87-101. 